\title{
No quiero tener hijos (as)... continuidad y cambio en las relaciones de pareja de mujeres profesionales jóvenes
}

\author{
Fernanda Chacón Onetto
}

Universidad Arturo Prat, Iquique, Chile

Email: fernanda.c.onetto@gmail.com

\section{Marcela Tapia Ladino}

INTE, Universidad Arturo Prat, Iquique, Chile

Email: marcela.tapia@unap.cl

\begin{abstract}
Resumen $^{1}$ : En un contexto de transformaciones culturales, económicas y sociales que han experimentado las familias chilenas y de diversos cambios en el marco de la modernidad, el presente artículo analiza las principales motivaciones que expresan un grupo de mujeres profesionales acerca de la decisión de no tener hijos (as). A partir del análisis de las experiencias biográficas y familiares, la decisión se presenta en estrecha relación con la conformación de relaciones de pareja más igualitarias en las que se entremezclan prácticas y representaciones que discuten los mandatos de género. Entre ellos los significados respecto del trabajo, la pareja, los hijos, el uso del salario y su distribución de tareas dentro del hogar, entre otros. A pesar del interés por construir relaciones más igualitarias, la opción por la no maternidad no asegura el fin de las desigualdades especialmente en relación al reparto de tareas al interior del hogar. Sin embargo, en el grupo estudiado se aprecia un constante interés por construir a una relación más equitativa y propender a una relación distinta a las que ellas conocieron en sus respectivas familias.
\end{abstract}

Palabras claves: No maternidad, relaciones igualitarias, género

\section{I don'twant to have children... Continuity and change in partner relationships of young professional women}

\begin{abstract}
In the context of the cultural, economic and social transformations experienced by Chilean families and various changes related to modernity, this article analyzes the main motivations expressed by a group of young professional women regarding the decision not to have children. Based on the analysis of family and life experiences, the decision is presented by them as being closely connected to the development of more equal partner relationships in which practices and representations that challenge gender mandates are intermingled. This includes aspects related to work, one's partner, children, income use and the distribution of household tasks, among others. Despite the interest in building more equal relationships, the decision not to have children does not ensures the end of inequalities, especially in relation to the distribution of household chores. However, the studied group presents
\end{abstract}


a constant interest in building a more equitable relationship and a move towards interaction that differs from the ones they experienced in their respective families.

Keywords: no maternity, equal relationships, gender

\section{Eu não quero ter filhos (as) ... continuidade e mudança nas relações de jovens mulheres profissionais}

Resumo: Num contexto de transformações culturais, econômicas e sociais que têm experimentado as famílias chilenas e das diversas mudanças no contexto da modernidade, este artigo analisa as principais motivações que expressam um grupo de mulheres profissionais sobre a decisão de não ter filhos (as). A partir da análise de experiências biográficas e familiares, a decisão a apresenta em estreita ligação com o estabelecimento de relações mais igualitárias em que se misturam as práticas e representações que discutem os mandatos de gênero. Incluindo os significados sobre o trabalho, o casal, crianças, o uso do salário e a distribuição de tarefas dentro do lar, entre outros. Apesar do interesse na construção de relações mais igualitárias, a escolha pela não-maternidade não garante o fim das desigualdades, especialmente sobre a divisão de tarefas dentro do agregado familiar. No entanto, no grupo pesquisado aprecia-se um interesse constante por construir uma relação mais equitativa e promover uma relação diferente daquela que elas tiveram em suas respectivas famílias.

Palavras-chave: Sem maternidade, relações igualitárias, gênero

$$
* * *
$$

\section{Introducción}

El presente artículo es el resultado de una tesis de pregrado cuyo objetivo fue analizar los factores socioculturales y biográficos involucrados en la decisión de no tener hijos (as) de un grupo de mujeres profesionales con pareja (todas unidas por convivencia) de Iquique y Santiago. Este estudio nos desafía a considerar dos aspectos centrales en el análisis: las relaciones de género y los cambios en la organización familiar como marco general en el caso de un grupo de mujeres profesionales ¿Cuáles son los factores que influyen en la decisión de no tener hijos (as)? ¿Qué aspectos de las biografías de las entrevistadas inciden en esta decisión? y si ¿Esta opción se traduce en mayor igualdad de género o procesos de emancipación femenina? Estas son algunas de las interrogantes que nos proponemos desarrollar en este manuscrito.

El estudio sobre los factores que motivan la decisión por no tener hijos (as) podría ayudar a explicar el descenso de la fecundidad que presenta Chile en los últimos años, el conocimiento de las transformaciones familiares y en las relaciones de género por las que transita la sociedad junto con los cambios en la posición social de las mujeres y de los hombres, entre otros aspectos (Dema, Sandra, 2006;Flaquer, 1999;Hooper, Wallace y Doehler, 2012). 
A partir de las transformaciones herederas de lo que Camps denominó la revolución silenciosa de las mujeres del siglo XX (1998), se pueden dilucidar distintos cambios en las expectativas y posición social de las mujeres como de la estructura y organización familiar (Olavarría, 2014). El modelo de familia burguesa que se configuró a partir de la revolución industrial y que fue tomando fuerza durante el siglo XIX y particularmente en XX se basó en la existencia de hombres proveedores (malebreadwinner) y mujeres amas de casa (Olavarría, 2014). Este modelo se constituyó por largo tiempo como un referente positivo de modelo de mujeres y hombres, el que sin embargo ha sido objeto de debate por la invisibilización de los distintos tipos de familias (Gonzálvez, 2015) y pérdida de valor de este paradigma en un escenario de transformaciones económicas y sociales (Tobío, 2005). No obstante, y como ha documentado la historia es preciso advertir que en el caso de las mujeres de clase trabajadora éstas desde siempre se vieron frente al desafío de compatibilizar el trabajo dentro y fuera de la casa en un contexto de precariedad laboral (Duby, Perrot, Thébaud, y Nash, 2006; Salazar, 1992). La diferencia hoy día es que éste desafío se ha extendido al grueso de las mujeres trabajadoras, superando la adscripción por clase. Hoy cada vez más el ingreso al mercado laboral está afectado por la necesidad económica, pero también por el deseo de independencia económica, especialmente entre mujeres jóvenes con mayores niveles de escolaridad (Díaz, Godoy, y Stecher, 2005).

Respecto de la organización familiar, los cambios en el modelo de familia nos remiten a transformaciones en las conductas vitales, especialmente de las mujeres. Al respecto, según el informe Anual de Population Reference Bureau se advierte una disminución en el número de hijos por mujer, la tasa global de fecundidad a nivel mundial ha pasado de 4,7 hijos por mujer en 1970 a un promedio de 2,5 hijos en la actualidad(Mensch, Sing, \& Casterline, 2005). De igual forma se registra un aumento en la edad de las uniones matrimoniales entre los años 1990-2001, sólo un 37,7\% de las mujeres encuestadas de entre 20 a 24 años en 51 países alrededor del globo estaba casada a los 18 años, cifra que aumenta a un 52\% entre las mujeres de 40 a 44 años de edad encuestadas en los mismos años. En tanto las mujeres encuestadas de entre 30 a 44 años de edad que habían contraído matrimonio a los 25 años supera el 85\% (Mensch et al., 2005).

Dentro de este amplio abanico de cambios en la constitución familiar y las relaciones de género surgen nuevos patrones de conducta reproductiva como es el caso de las mujeres con parejas que no desean tener hijos (as). Según un informe elaborado por el Centro de Microdatos de la Universidad de Chile en base a la Encuesta de Protección Social el año 2006, del total de las mujeres en edad fértil que no tienen hijos (as), el 47\%, no desea tenerlos a futuro, lo que equivale a 275.000 mujeres(Altomonte y Albagly, 2010). Si bien se trata de un fenómeno poco significativo en términos estadísticos, es expresión de una transformación paulatina que nos motiva a preguntarnos si se trata o no del tránsito hacia mayores niveles de autonomía y empoderamiento por parte de las mujeres. Ello porque la decisión de no tener hijos (as) es una muestra del alejamiento del mandato 
tradicional que define inequívocamente a las mujeres con la madre y a la maternidad como la mayor expresión de lo femenino, es decir, que "toda mujer no sólo es madre en potencia, sino también en necesidad” (Saletti, 2008, p. 173). Por tanto interesa indagar por los factores que motivan esta decisión y si se trata de cambios que propician relaciones de género más igualitarias o se trata más bien de un acto de resistencia al mandato de maternidad y la responsabilidad que implican los hijos e hijas.

De la mano de las transformaciones señaladas se registran cambios en el sistema de valores y el estilo de vida de las personas. Se experimenta un sentido de la privacidad alejado de lo público, como un 'orden natural' que permite a las personas mantener un modelo doméstico-privado en función de sus intereses económicos y afectivos(Parella, 2003). En definitiva, se asiste a un cambio de mentalidad en los que valores como la autonomía y la independencia económica adquieren una centralidad cada vez mayor entre hombres y mujeres, lo que ha ido transformando decisivamente las identidades femeninas. Así, las mujeres que entraron al siglo XXI son muy diferentes a aquellas que inauguraron el siglo XX y hoy las oportunidades se han ampliado y se han extendido los horizontes posibles. Sin embargo, se reconoce que la desigualdad y la inequidad no han desaparecido y se mantienen nudos críticos que demuestran la prevalencia del patrón de dominación patriarcal ${ }^{2}$.

La investigación desarrollada fue de tipo cualitativo y el diseño de investigación elaborado fue de carácter narrativo ${ }^{3}$, es decir, la recolección de la información se obtuvo con la técnica de entrevistas en profundidad. Las mujeres que conformaron el corpus de la investigación se ubican entre los 29 a 39 años de edad, todas mantenían relaciones de pareja heterosexuales, eran profesionales y mantenían alguna actividad económica remunerada. Un requisito fundamental era que la decisión de no tener hijos (as) debía expresarse por las informantes como definitiva, al menos al momento de las entrevistas, no importando si en un futuro esa opción pudiera presentar variaciones. El objetivo fue reconstruir las historias de vida de las mujeres con el afán de identificar los factores que motivaron la decisión de no tener hijos (as) por parte de las entrevistadas.

Se realizaron un total de 14 entrevistas a 4 mujeres. Para ello se programó 1 entrevista semanal con cada participante cuya extensión bordeaba 1 hora y media aproximadamente Este procedimiento se extendió durante 4 semanas.Las entrevistas realizadas se elaboraron en base a categorías analíticas que consideraron las características de la familia de origen, los hitos de la vida familiar y la historia que cada mujer había construido en su vida con distintas parejas, donde se incluyó las responsabilidades en el reparto de tareas al interior del hogar. Esta última dimensión presenta una importancia particular, puesto que entrega algunas luces sobre la forma en que se articula la desigualdad en la familia, unidad que actúa como un medio de expresión de la individualidad. Luego de la primera entrevista se incluyeron categorías de análisis emergentes entre ellos los significados en torno a la sexualidad, el trabajo y la educación. Todas las entrevistadas tenían 
parejas al momento de realizar las entrevistas, la mayoría superaba los tres años de relación y se encontraba conviviendo, por lo que ya habían establecido un proyecto de vida en común. En general las mujeres entrevistadas se ajustaban al modelo de "parejas de doble ingreso", en unos casos había similitud en los sueldos y en otros ellas tenían mayores ingresos que su parejas(Dema, 2006).

El artículo se organiza de la siguiente forma, la primera parte contiene antecedentes generales del fenómeno de las mujeres que voluntariamente no quieren tener hijos (as) en Europa y América Latina. Asimismo se aborda el marco teórico basado en los aportes del feminismo en los estudios de género y el desarrollo más recientes sobre los estudios de las familias,así como el vínculo con los cambios en las relaciones de género y en la organización familiar. En la segunda parte se pasa al análisis de las entrevistas, los hallazgos, para lo cual se consideró los argumentos y decisiones que coinciden con el relato de las entrevistadas. Se consideran las razones de la decisión en el valor que tiene para las entrevistadas la autonomía en sus vidas, luego aquellas que se anclan en la experiencia adquirida durante la adolescencia y juventud. Luego se revisan los acuerdos tomados en la pareja y los pactos fundantes que concretan la decisión y el afán de conseguir relaciones de género más equitativas. La tercera parte corresponde a las continuidades y cambios en las relaciones de género a partir de la decisión de no tener hijos (as) y el lugar que ocupan los recursos económicos y la división sexual del trabajo al interior de la relación de pareja.

\section{La opción por no tener hijos (as) Childfree, NoMo o Dinks los cambios en las relaciones de género y las formas de hacer familia}

\section{Antecedentes generales}

La disminución progresiva de las tasas de fecundidad y fertilidad alrededor del mundo ha suscitado creciente interés en el público, en los medios de comunicación y en el ámbito académico. En este sentido, han surgido diversas siglas para denominar a las mujeres y sus parejas que voluntariamente no quieren tener hijos (as); Childfree, NoMo, Dinks, entre otras. ${ }^{4}$ En Europa las cifras son reveladoras: La tasa global de fecundidad mediaha llegado a 1,5 hijos por mujer (Baird et al., 2010). De acuerdo al Microcenso realizado en Alemania el año 2012, alrededor del 22\% de las mujeres de entre 40 y 44 no ha tenido hijos. En la República Federal, las mujeres sin hijos llegaron al 23\% en el mismo año (Centro Alemán de Información, 2013). Según el UnitedStatesCensus Bureau, para el año 2014 las mujeres de entre 40 y 44 años de edad que no han tenido hijos llega al 15.3\% en Estados Unidos (USAID, 2014).

Latinoamérica también ha experimentado un descenso de la fecundidad. En 1950 la tasa global de fecundidad en Latinoamérica era de 5,9 hijos 
por mujer, cifra que se mantuvo estable hasta 1965, donde se observa un descenso constante hasta la actualidad (CEPAL, 2008, p. 11). Desde mediados de los años 60 hasta el año 2010, la TGF en el continente se redujo en un 59\%. Las disparidades en cuanto a los patrones reproductivos de las mujeres en la región se deben principalmente a las diferencias entre el desarrollo de los distintos países (CEPAL, 2008). Ahora bien,es preciso indicar que la disminución de la maternidad entre mujeres mayores, es decir, entre 30 a 49 años, es la que ha impulsado en su mayoría la reducción de la fecundidad en Latinoamérica, seguida por las mujeres de entre 25 a 29 años (CEPAL, 2008).

En Brasil y Uruguay - países en transición demográfica avanzada - el porcentaje de mujeres de entre 45 a 49 años que no han concebido hijos llega a 12 y 10 por ciento respectivamente para el año 2010 (INE, 2014). En Chile, la tasa global de fecundidad ha disminuido en un 65\% en 42 años; desde el año 1962 hasta el 2004 (INE, 2006). El mayor aporte porcentual a la fecundidad total para el año 2011 en el país lo realizan las mujeres de entre 25- 29 años de edad, seguidas por las mujeres de entre 30 - 34 y 20 - 24 años de edad, grupo que ha sufrido una disminución importante desde 1990 (INE, 2011). Si bien en Chile y Latinoamérica la fecundidad de las adolescentes y jóvenes (15- 19 años) tiende a disminuir en el tiempo, en el país la tasa de natalidad de este grupo etario se mantiene alta (ONU, 2015).

Un reporte emanado del Centro de Microdatos de la Universidad de Chile indica que en el país a medida que aumenta la edad de las mujeres, aumenta también el porcentaje de ellas que considera no tener hijos (as) en el futuro, así, si dentro del tramo de mujeres de entre 20 - 24 años de edad sólo un 21,96\% no tiene pensado tener hijos (as), esta cifra llega al 92.73\% en las mujeres de 45 a 49 años de edad (Quijada, 2010). Este mismo estudio señala que las mujeres en edad fértil que no tienen hijos (as) -y que no desean tenerlos en el futuro- llega a un 46,9\%, frente a un $46,3 \%$ que si lo considera (Quijada, 2010).

Con respecto a las causas de la reducción de la fecundidad como fenómeno global, específicamente en el caso de las mujeres que deciden no tener hijos (as), está estrechamente vinculada a su inserción al mercado laboral, al aumento en los niveles de renta y los niveles de escolaridad $^{5}$ (Cavenaghi, 2013; Centro Alemán de Información, 2013). Estos cambios han impactado a las familias, en tanto han favorecido "el cuestionamiento del arquetipo tradicional de distribución de roles laborales y familiares” propiciando así la transformación paulatina de los roles de género dentro de ellas (Caamaño, 2010, pp. 197-198). Como indica Jelin (2006) bajo el contexto actual "el valor de los hijos y la vida familiar gradualmente cambia de lugar”, constituyéndose cada vez más en una prioridad el acceso a mayores niveles de educación y mejores oportunidades laborales (p.11)

\section{La mirada desde el feminismo y las nuevas formas de hacer familia}

Para comprender las razones que llevan a algunas mujeres a optar por no tener hijo/as es central incluir en el análisis los aportes de la teoría 
feminista a las Ciencias Sociales en general y el debate actual sobre las familias en particular. Respecto de lo primero es ampliamente conocida la capacidad explicativa de la perspectiva de género en tanto propicia un entendimiento generizado(Acker, 1990; Stacey \& Thorne, 1985), es decir, que el género como parte de todos los aspectos de la vida humana explica y da sentido a una realidad vivida de manera diferente, tanto si se trata de hombres o de mujeres. El hecho de desnaturalizar lo femenino y lo masculino e irracionalizar la sujeción de las mujeres a espacios normativos(Molina, 2003, p. 128), permite comprender los cambios en las relaciones sociales y la subversión normativa que implican dichas transformaciones (Valcárcel, 2009).

Es indudable que especialmente a partir de las luchas feministas se advierten cambiosdurante el siglo XX en Occidente, especialmente respecto de la situación y posición de las mujeres en las sociedades.Los aportes de la inclusión del feminismo en las Ciencias Sociales cuestionó la rigidez con las que se interpretaban las identidades de género, y los logros del movimiento feminista se tradujeron en un mayor acceso de las mujeres a la educación y al mercado de trabajo, en cambios culturales, demográficos, y en las mentalidades y las familias en Occidente (Duby et al., 2006)lo que permitió y ha permitido a las mujeres "definirse y ser definidas en otros términos distintos a los de la maternidad” (Hird \& Abshoff, 2000, p. 351).Sin embargo, el avance de las mujeres y de las luchas feministas no implica necesariamente un cambio radical en las relaciones de género puesto que las transformaciones no van siempre en una sola dirección. En este sentido aunque el patriarcado se presenta como una patrón inmutable (Bastos, 1999) que experimenta lentos cambios, se distinguen procesos a nivel micro, de acuerdo a negociaciones patriarcales (patriarchalbargains) (Kandiyoti, 1988), especialmente de parte de las mujeres, en pos de conseguir cuotas de poder o ciertos niveles de autonomía dentro de la unidad familiar. Los pactos fundantes en las relaciones de pareja - definidos como una serie de reglas y límites tácitos y explícitos destinados a definir el compromiso adquirido en la vida en común- forman parte de estas estrategiasque buscan conducir, en algunos casos, a formas de interacción más equitativas y en otros, a negociaciones que no cuestionan necesariamente la relación asimétrica de la pareja, pero que suponen un tránsito hacia relaciones más democráticas(Tapia, 2010).

A partir de las conquistas feministas la autonomía se vuelve un valor central para la conformación de las identidades ${ }^{6}$ de las mujeres, sobre todo para quienes deciden no tener hijos (as). En este trabajo adoptaremos la definición de autonomía de Marcela Lagarde(1999), quien supera la visión atomizada de la autonomía kantiana vastamente criticada por las feministas(Code, 1991; Jaggar, 1985). La autora concibe a la autonomía como la capacidad de definición propia, de existir por sí mismas y en tanto tal, como un estado alcanzable que se construye socialmente a través de procesos vitales, económicos, culturales y sicológicos(Lagarde, 1999). Como tal depende de condiciones estructurales, las que se relacionan con las oportunidades y con la garantización de las libertades individuales, de expresión, asociación, de culto, de movimiento, etc., en un sentido político que 
permitan a las mujeres decidir sobre lo que desean ser y hacer (Mackenzie, 2014). Por tanto la autonomía es social porque involucra a las personas sus ámbitos sociales y sus relaciones y en este sentido es un "pacto social" puesto que tiene ser "reconocida y apoyada socialmente, tiene que encontrar mecanismo operativos para funcionar” (Lagarde, 1999, p. 7). Las luchas feministas han sido claves para promover la autonomía de las mujeres porque ésta requiere de una serie de condiciones sociales, jurídicas y económicas para que pueda desenvolverse, pero también supone una conciencia por parte de las mujeres para propender a alcanzarla.

Es diferente de la independencia, concebida como una forma de relacionarse (Friedman, 2014), puesto que a las mujeres históricamente se les ha limitado la independencia, en tanto dependen o han dependido de otros, económica, jurídica y socialmente, pero no necesariamente se les ha anulado la potencialidad de autonomía (Lagarde, 1999). Así, la independencia económica, esto es, "no depender del apoyo financiero de otra persona" (Friedman, 2014, p. 56) es considerada como un aspecto necesario más no suficiente para la autonomía, ya que como indica Dema, "ganar dinero no implica un uso autónomo del mismo”(Dema, 2006, p. 67). Por ello es que investigaciones que escudriñan la relación entre la autonomía y el trabajo extradoméstico han señalado que son los significados en torno al trabajo como una actividad útil y beneficiosa, y la centralidad del mismo en los proyectos vitales, los que estarían involucrados en el aumento de los niveles de autonomía en las mujeres, sobre todo para las que poseen mayores niveles de escolaridad (García y Oliveira, 2007).

Con todo, los avances en las conquistas feministas y los cambios en la posición social de las mujeres no significa que la organización patriarcal cambie radicalmente o que los varones pierdan preeminencia. Para comprender la dificultad que las mujeres experimentan para construir relaciones más equitativas con sus parejas nos parece útil la inclusión de la noción de micromachismo. Este concepto alude "al abanico de maniobras interpersonales que realizan los varones para intentar mantener su dominio y su supuesta superioridad sobre la mujer objeto de la maniobra; reafirmar o recuperar dicho dominio ante una mujer que se 'rebela' por 'su' lugar en el vínculo; resentirse al aumento de poder personal o interpersonal de una mujer con la que se vincula o aprovecharse de sus poderes”(Bonino, 1996). Se trata en general de conductas sutiles, cotidianas e invisibles que buscan mantener la posición de poder de los varones.

Los estudios históricos y antropológicos más recientes sobre familias han transitado desde la preocupación por ellas a la discusión de la conceptualización y lo que se entiende por familias. De modo que se ha pasado del interés por los distintos tipos de familias a la adopción de una noción más precisa que da cuenta de la variedad de formas que adopta esta institución social. Hoy la discusión se centra en lo que se denomina formas de "hacer familias", es decir, de las “configuraciones de parentesco, distribución de roles y tareas, concepciones parentales y filiatorias” (Salvo y Gonzálvez, 2015, p. 41). Se trata de una diversidad de formas familiares que 
ponen en entredicho las nociones de viejas y nuevas familiasy que han explicitado y visibilizado las diferencias entre lo biológico y lo social y el mayor margen de elección que tienen las personas para construir distintas formas de familia en la actualidad(Gonzálvez, 2015). Este debate discute la asociación entre "la sexualidad, la procreación, la alianza y la filiación, cuestionando así el modelo biparental -padre/madre- heterosexual dominante en la visión cultural del parentesco occidental” (Salvo y Gonzálvez, 2015, p. 41). De modo que los fenómenos como la monoparentalidad femenina y masculina y las distintas formas de fertilización asociadas o la opción voluntaria a no tener hijos (as), son algunos de los aspectos más notorios sobre las formas familiares que observamos hoy (Hooper, Wallace, Doehler, \& Dantzler, 2012).

De acuerdo a las transformaciones señaladas, la familia nuclear patriarcal o la llamada familia conyugal ha sido fuertemente discutida, en tanto se ha puesto en entredicho su universalidad como patrón familiar y debatido los roles que hombres y mujeres realizan así como los valores claves que rigen su permanencia (Hooper et al., 2012). En la actualidad el vínculo entre los cónyuges propende hacia relaciones más igualitarias y democráticas con mayor poder de negociación por parte de las mujeres y los hijos (as), con cada vez mayor responsabilidad del padre en su crianza ${ }^{7}$. Estos cambios van de la mano de una apertura de las formas de organización familiar, las que varían según estructura - familias biparentales sin hijos(as), uniparentales con o sin hijos (as) con jefatura femenina, extensas biparentales o monoparentales, compuestas (Ariza y Oliveira, 2001; Hooper et al., 2012; Jelin, 2006; Olavarría, 2014).

\section{No quiero tener hijos (as)... Factores explicativos de la decisión y representaciones en torno a la maternidad}

\section{El valor de decir por sí mismas y disfrutar la vida}

A partir del análisis de las entrevistas y de acuerdo a una perspectiva diacrónica se identificaron los factores que sustentan la decisión por la no maternidad voluntaria, donde se incluyen significados y representaciones en torno a la maternidad detectada en la historia de vida de las entrevistadas. En este sentido uno de los principales motivos que impulsa la decisión de no ser madres en las participantes se relaciona con la necesidad de resguardar un valor central a nivel identitario, esto es, la autonomía, o en términos prácticos, la posibilidad de decidir sobre sus vidas y definir sus proyectos personales (Lagarde, 1999). Consideran que la autonomía y la maternidad están en contradicción, que son incompatibles debido principalmente a los significados que las mujeres del estudio le confieren a esta última: como una actividad que les imposibilita decidir de acuerdo a sus propios deseos, a lo que quieren para sí, ya que ubordina las aspiraciones y anhelos de la mujer a las necesidades del hijo (a). Así, de acuerdo a las entrevistadas, el desarrollo de los proyectos personales deja de erigirse 
como una prioridad en la vida de las mujeres que optan por la maternidad, quienes centran su tiempo y esfuerzos en el cuidado y bienestar de los hijos (as). Estos últimos dependen completamente de la madre, sobre todo en las primeras etapas de vida, lo que hace de la maternidad una actividad altamente demandante. Así la necesidad de establecer las condiciones propicias que les permitan decidir sobre sus proyectos vitales, disponer de tiempo y estar físicamente habilitadas para la conquista de aspiraciones educacionales, profesionales, los que en el caso de las mujeres del estudio se orientan a la consecución de estudios de postgrado y a la consolidación de sus carreras. Así, por ejemplo, una de las informantes se trasladó de Santiago a Iquique en busca de mejores oportunidades laborales, con mayores niveles de renta y mejor ambiente laboral.

"Yo creo que ese tema como de dependencia, de lo que significa, no sé si quiera hacerme cargo de una persona porque al final eres mamá y hasta que te mueres tenis que estar pendiente. Yo lo veo en mi tía: mis primos ya boludos y mi tía ahí. Por ejemplo en estos momentos si me sale una beca y me tengo que ir a Argentina: ¿Qué voy a hacer si tengo una guagua?, ¿dónde la dejo? Yo ahora digo: me pagaron y me quiero ir a Machupicchu, agarro mis cosas y me voy, pero con una guagua ya es diferente” (C.B, Socióloga, 37 años, Iquique).

"Yo creo que disfrutar la vida es no echarse encima responsabilidades que uno puede eludir y tener la libertad para hacer lo que uno quiere en el minuto que quiere, o sea, no me refiero a mandarte a cambiar así a las 12 del día porque te dio la tontera en la oficina, me refiero en términos generales, así como lo que yo quiero hacer en la vida, poder hacerlo y no tener que decir oye, sabí que en realidad a mí me encantaría, pero pucha, en realidad no puedo porque como tengo hijos, tengo que preocuparme del colegio, con quien los dejo, en realidad no puedo darme el lujo de ir a dar la vuelta al mundo y pedir un año sabático porque en realidad cómo mantengo a mis hijos ese año, cachai? Poder hacer lo que yo quiera, o sea, no tener nada que me amarre”(M.M, 39 años, Contadora Auditora, Iquique).

De esta manera, la autonomía en ellas se relaciona fuertemente con un sentido de libertad expresado en la capacidad de decisión y planificación de sus proyectos biográficos. Dicha planificación indica una orientación activa en la constitución de sus historias de vida (Lagarde, 1999). Dentro de las proyecciones y las expectativas que poseen las mujeres acerca de su futuro se encuentra la decisión de no tener hijos (as), la cual se expresa como un "proceso" mentado e introspectivo: “Creo que es un proceso reflexivo que se centra en el ser mujer y tomar conciencia de tu cuerpo” (C.B, Socióloga, 37 años, Iquique).

Ahora bien, con respecto a las representaciones en torno a la maternidad, las entrevistadas disocian la sexualidad de la reproducción y la maternidad de lo femenino. Para ellas la maternidad es una opción racional, pero no un mandato "natural” y por tanto las mujeres pueden decidir si 
desean o no tener hijos (as) y controlar la opción. Como señaló una de las entrevistadas:

"Si fuéramos animales no racionales sería un instinto de reproducción porque al final los animales tienen sexo, copulan para poder reproducir su especie, pero nosotros somos animales racionales, entonces no creo en el instinto de la reproducción. Yo creo que uno claro, quiere tener su affair o te dan ganas de tirar con alguien, pero eso no va a ser para reproducirte porque tu podís tomar pastillas o cuidarte con condones, entonces no lo veo de esa manera” (C.B, Socióloga, 37 años, Iquique).

\section{La historia familiar y los modelos de pareja como experiencias explicativas de la decisión de no tener hijos (as)}

A los significados construidos en torno a la maternidad, la sexualidad y la autonomía se suman otros aspectos que tienen que ver con la historia familiar y la vivencia personal. En la mayoría de los casos estudiados se reconocen experiencias de parentalización temprana durante la niñez y juventud, es decir que las funciones socialmente definidas para un padre o madre, - los roles-, son asumidos por los hijos (as) dentro de la familia, reemplazando así las responsabilidades tanto afectivas como instrumentales que delimitan la parentalidad (Hooper, et al., 2012). Esto implica un cambio de roles entre los miembros de la familia, una distorsión de las fronteras entre las distintas generaciones que la componen y una "sobrecarga de responsabilidad al niño o adolescente con respecto a su rol y a la etapa del ciclo vital en la cual se encuentra” (Barbosa, Segura, Garzón, y Parra, 2014). A lo que se suma un modelo de pareja de los padres caracterizada por la desigual y agudizada división sexual del trabajo al interior de la familia. Ambos hechos son los motivos centrales que las entrevistadas identifican como argumento para no tener hijos (as) y buscar relaciones de pareja más igualitarias o simétricas sin la carga que supone tener hijos (as). Durante la relación no sólo los acuerdos de la pareja, los pactos fundantes son relevantes, sino también una serie de estrategias de resistencia que propician la mantención de ese ideal como veremos más adelante.

Un elemento que se repite en el relato como elemento decisivo en la opción por la no maternidad fue la sobrecarga de actividades domésticas y de cuidado dentro de sus respectivas familias de origen a temprana edad. Este aspecto resultó invariable en el relato analizado: todas las mujeres habían atravesado por estas experiencias con anterioridad, la mayoría de ellas durante su infancia, asumiendo la responsabilidad del cuidado de sus hermanos menores, y, para algunas de ellas, el de sus padres durante la adolescencia. Muchas veces dichas responsabilidades se extendían a la realización de tareas domésticas, por ejemplo lavar, planchar, y mantener el orden del hogar en ausencia de los padres durante sus jornadas laborales. Las labores de cuidado iban desde ayudar a sus hermanos en sus deberes escolares, asegurar su alimentación diaria, hasta hacerse responsables por su seguridad. 
Aquella experiencia fue clave en la decisión de las mujeres de no tener hijos (as). Para las informantes significó una prueba temprana de las tareas que implica la maternidad y por extensión del trabajo doméstico y lo limitante que puede ser para la consecución de proyectos personales (Ajenjo y García Román, 2010). Aquellas vivencias han ayudado a construir los significados de la maternidad como un impedimento para el desarrollo personal y profesional:

"[Mi hermano menor] influye un poco en que yo no quiera ser mamá. Muchas de las etapas de crianza, de hacerme cargo de una guagua, de vivir la maternidad, yo siento que ya la viví un poco con él. Yo era la hermana grande, mis papás se separaron al año de que el nació, entonces mi mamá trabajó toda la vida, entonces nos tuvimos que hacer mucho (sic) cargo mi hermana y yo de él, de ir a las reuniones de apoderados, de ayudarlos con las tareas” (M.C, Ingeniera Civil Industrial, 33 años, Santiago).

Otro elemento decisivo en la decisión de no tener hijos (as) se relaciona con el modelo de pareja aprendido dentro de sus familias de origen. En general las informantes han sido socializadas bajo el alero de un modelo de pareja patriarcal, donde sus madres presentaban alta adherencia al rol femenino tradicional, situación que favorecía relaciones desiguales entre los cónyuges. En las relaciones cultivadas en la familia primaba el servilismo y la subordinación por parte de las mujeres y la anteposición de los intereses de otros antes que los propios. Las constantes peleas en el hogar, la mayoría iniciadas por parte de las madres hacia sus esposos, se constituyeron para las informantes en las razones de los altos niveles de amargura e infelicidad en sus madres. Esta experiencia las motivó a evitar replicar vivencias parecidas y a generar relaciones diferentes durante su adultez cimentadas en el respeto por la individualidad y autonomía personal:

"En general en mi vida amorosa no he tenido relaciones desastrosas. Yo creo que eso me pasó porque ví a mi mamá, entonces como (sic) que yo soy todo lo contrario, y creo que es por eso, yo lo asumo así, creo que es porque ví a mis papás discutir y en el fondo igual las cosas te afectan... si yo hubiera sido mi mamá y con las lucas (sic) que ella ganaba, yo echo cagando (sic) a mi papá, pero mi mamá era muy dependiente de mi papá. Generaba esas relaciones” (C.B, Socióloga, 37 años, Iquique).

Entre los factores que las entrevistadas identificaron como el origen de los conflictos se encontraban la distribución desigual de poder dentro de su núcleo familiar:

“yo veía en ella [la madre] la personificación de la frustración. Era una mujer frustrada, que no hizo lo que quiso, que los hijos pa (sic) ella fueron un desastre porque le coartaron su vida, se tuvo que quedar al lado de mi papá toda la vida porque tenía hijos con él, 
porque dependía económicamente de él. De chica yo solo veía una mujer irracional” (M.M, 39 años, Contadora Auditora).

Esta situación propició en ellas la generación de nuevos significados en torno a la pareja, especialmente en torno a una relación más democrática y de compañerismo:

"Para mí la pareja es un compañero, una persona que te apaña (sic), que está contigo, que rema pal (sic) mismo lado que tú en muchas cosas. Creo que la construcción de pareja se hace entre los dos sin invadir al otro" (C.B, Socióloga, 37 años).

\section{La decisión de no tener hijos (as) como pacto fundante de la relación de pareja}

Los factores que se configuran a partir del establecimiento de vida en pareja es el segundo eje explicativo de la decisión de no tener hijos (as) de las entrevistadas. En general en la mayoría de las relaciones afectivas matrimonios o parejas- se establecen acuerdos o pactos fundantes, conversados y discutidas por ambos (Tapia, 2010). Entre los acuerdos tácitos o implícitos, se encuentran las que definen la forma en que se relacionan con otras personas, el rol que deben cumplir el uno con el otro y los valores o significados que son importantes de preservar en la relación, entre los aspectos más significativos. Dentro de los pactos fundantesde la relación se encuentra la decisión de no tener hijos (as), decisión conversada y aceptada porlas respectivas parejas. Esta forma de relacionarse está basada en la negociación y la comunicación que se ajusta a las expectativas sociales del cómo debe desarrollarse el vínculo amoroso en plena modernidad (BeckGernsheim, 2003). Lo mismo sucede con el matrimonio, la mayoría de las mujeres indicaron que este no se encontraba dentro de sus planes, ni el de sus parejas, ni a corto ni largo plazo, decisión también pactada entre ambos.

Así los pactos fundantes establecidos por las entrevistadas en su relación de pareja estaban definidos de acuerdo a la expansión de los valores centrales de la modernidad, entre ellos la autonomía, es decir la capacidad de ser por sí mismas y la posibilidad de actuar según los propios deseos. Esto quiere decir que están en función del reconocimiento de las necesidades personales y de los deseos de carácter subjetivo. Dichos elementos implican la posibilidad de romper los vínculos afectivos cuando el amor de la pareja se acaba o cuando los conflictos en ella vulneran el bienestar personal. Esto cuestiona la idea del amor como un vínculo imperecedero y por tanto la expresión "para toda la vida" deja de tener sentido ${ }^{8}$ (Jelin y Paz, 1991). Por ello es que las separaciones y uniones pasan a ser concebidos como un proceso normalizado para las entrevistadas, o como parte de las reglas del juego (Beck-Gernsheim, 2003).

Ahora bien, la tendencia discursiva de las mujeres entrevistadas estuvo marcada por el ideal de pareja simétrica, es decir, una relación donde los roles de género tradicionales han sido relativamente modificados en pro 
de relaciones más igualitarias (Dema, 2005). Uno de los indicadores que hizo posible identificar el ideal de pareja simétrica en los discursos de las entrevistadas fueron los significados que las informantes les atribuían a sus parejas. La mayoría de ellas se refería a ellos como “compañeros” en igualdad, "un par”, reafirmando siempre su independencia con respecto a ellos, como individuos que comparten una vida en común, conservando sus individualidades y particularidades. La necesidad de negociar y conversar para lograr puntos de encuentro o arreglos momentáneos o duraderos, constituye en sí una estrategia que concuerda con el ideal de pareja igualitaria, aspecto altamente valorado y utilizado por las informantes (Dema, 2005).

\section{La opción por la no maternidad, continuidad y cambio en las relaciones de género}

\section{La búsqueda de la igualdady los recursos económicos}

En este contexto una dimensión explorada en las entrevistas fue la consecución de recursos económicos como elemento que favorece la equidad en las relaciones de pareja. La mayoría de las entrevistadas dieron un gran valor al trabajo remunerado fuera de la casa y a ganar su propio dinero:

"El trabajo es fundamental en mi vida, básico, porque significa que te genera lucaspo (sic), y tú las lucas las necesitas para vivir, desgraciadamente la sociedad que nosotros tenemos necesitamos independencia económica, y en la medida que tu logras tener ciertos flujos de ingresos puedes tener esa independencia económica y sobre la independencia económica vienen todas las demás independencias. Me carga la dependencia. Me gusta poder manejar las situaciones, no que mi vida la maneje otra persona o que mis aspectos dependan de otras personas” (M.M, Contadora Auditora, 39 años).

Si bien todas las informantes se encontraban trabajando al momento de realizar las entrevistas, y algunas inclusive ganaban más dinero que sus parejas, ello no se tradujo en un reparto igualitario de las tareas domésticas. Estudios como los llevados a cabo por Ajenjo y García Román (2010)o Dema (2005), indican que efectivamente el trabajo remunerado deja en mejor posición a las mujeres que trabajan que las que no lo hacen. Por ejemplo, la aportación de los hombres al trabajo no remunerado es significativamente mayor en las parejas de doble ingreso que en los modelos tradicionales (Ajenjo y García Román, 2010: 20) pero el factor que adquiere mayor relevancia para la conquista de la equidad dentro del hogar es el significado que las mujeres le atribuyen a su actividad remunerada. Las entrevistadas asumen una identidad como mujeres independientes económicamente y empoderadas en el uso del propio sueldo, en sus cuerpos, etc.-, valores que ellas mismas expresaron como cruciales, tanto en su constitución individual como en las relaciones que entablan con otros: 
“yo creo que el trabajo, por lo menos como mujer, -no sé cómo lo verán los hombres-, te da independencia, por ejemplo, a mí no me gusta depender de mi pololo, a mi me gusta tener mi independencia económica, o sea, saber si yo quiero tirar toda la plata a la basura, lo voy a hacer y nadie me va a decir ninguna cuestión, por eso es importante pa' (sic) mí el trabajo, porque creo que te genera independencia económica y eso te genera independencia para hacer lo que tu quieras” (C.B, Socióloga, 37 años).

Pese a ello, fueron varias las situaciones encontradas que indican la existencia de una relación de poder desigual con respecto a sus parejas alejada del ideal igualitario declarado. Es preciso recalcar que, de acuerdo a las formas que adopta aquella desigualdad y los mecanismos que la mitigan - como el valor de la autonomía y su condición de trabajadoras y co proveedoras del hogar-, es que la desigualdad existente no las hace más dependientes de sus compañeros. Así, debido al ideal de pareja simétrica, y los mecanismos económicos que conlleva, es que el dinero que sus parejas ganan no es percibido como un mecanismo legitimador de la desigualdad, en tanto se difumina la imagen del hombre como proveedor, ello en parte porque ambos solventan los gastos del hogar que tienen en común.

En las entrevistas se advierte la importancia que tiene para las mujeres el trabajo remunerado y la administración de sus propios recursos. En este sentido, y a partir de los de las conquistas feministas, se pensó en el carácter liberador del dinero en las manos de las mujeres. Aunque hoy se reconoce su importancia para favorecer procesos de autonomía personal, la independencia económica no siempre ha sido una garantía o se ha traducido de manera directa en más poder para ellas. Distintos estudios muestran que la consecución de recursos económicos es una condición necesaria, pero no suficiente para alcanzar procesos de autonomía (Dema, 2005; Vogler, 1998).

Vogler y Pahl(1999) establecen una tipología que es útil para el análisis. El primero es el sistema de dinero común y administración conjunta en el que la pareja administra los recursos producidos. Una segunda forma, es el sistema de dinero común y la administración por parte de los hombres, en el cual estos administran todo el dinero de la pareja. El tercer modelo, corresponde al sistema de dinero común y la administración por parte de las mujeres, que como el caso anterior administra todo el dinero de la pareja. Una cuarta modalidad corresponde a la administración de una cantidad asignada, caso en que las mujeres sólo gestionan una parte del dinero que corresponde al dinero del hogar. El quinto modelo corresponde al sistema de administración separada, en el que tanto los hombres como las mujeres administran su propio dinero y cada uno de ellos se hace cargo de los diferentes gastos del hogar.

En el caso analizado la organización económica del hogar más utilizada entre las entrevistadas fue el llamado “fondo común” en el que cada parte aporta la misma cantidad de dinero que el otro, dejando el resto de sus 
sueldos libres para ser gastado como él o ella lo prefieran. Bajo este acuerdo económico los hombres y las mujeres son proveedoras del hogar e igualmente dueños de sus propios sueldos. Sin embargo, de quedar un delta de recursos esto queda a libre disposición de cada uno. Por tanto se trata de un régimen común, pero con un margen de independencia para el manejo de los recursos. Otra modalidad, aunque menos utilizada, fue la de compartir el uso de las cuentas corrientes, donde el dinero que ambos recibían el salario se conformaba como un "gran fondo" en el que estaba permitido utilizar el dinero del otro como se estimara conveniente.

Con respecto al salario, las entrevistadas expresan enfáticamente el valor que tiene el poder controlar los recursos que ellas mismas ganaban. Esta idea de ser propietarias de su sueldo se daba sobre todo en las entrevistadas cuyos acuerdos se alineaban con el llamado "fondo común". El uso y manejo del dinero ganado, luego de contribuir a la par con el mantenimiento del hogar, era decidido sólo por ellas, siendo de su libre disposición. Este representa otro cambio importante que demuestra la transformación paulatina de los roles designados para hombres y mujeres porque en general los hombres han sido socializados como individuos independientes, con derecho para manejar el dinero ganado libremente (Coria, 1991). El dinero ha funcionado históricamente como un elemento legitimador de la desigualdad en los hombres, en tanto a través de él ejercen su autoridad y control tanto de las mujeres como del hogar.

\section{La división sexual del trabajo doméstico. Resistencias y estrategias}

Cuando aludimos la división sexual del trabajo, entendemos de manera general, el reparto de tareas y responsabilidades de acuerdo al género (Amorós, 1995). Desde la antropología ha existido un amplio debate acerca del origen de la división del trabajo (Moore, 2004), sin embargo lo que interesa relevar es cómo se articula la asignación de tareas diferenciadas de acuerdo al género y la decisión de no tener hijos (as) y cómo cambian o no cambian las prácticas de género asociadas a dichos mandatos. En las sociedades occidentales ese reparto señala que las mujeres tienen como responsabilidad principal el cuidado de los hijos (as) y la realización de las labores domésticas y los hombres la realización de actividades fuera del ámbito doméstico, como el trabajo productivo y la provisión económica, como aspectos más relevantes. Se trata de tareas diferenciadas en el marco de una relación de poder asimétrica en el cual el trabajo desempeñado por las mujeres es menos valorado (Bosch y et al., 2003) .

Pese a que prácticamente todas las informantes sentían un rechazo por las labores domésticas, la mayoría de ellas se llevaba la mayor carga de trabajo al interior del hogar. Gran parte de las desigualdades se encontraban invisibilizadas por las mismas informantes por lo que les resultaba difícil asumir la desigualdad de dicho reparto debido al anhelo por construir rela- 
ciones más igualitarias. Lo que se advierte en los relatos sobre las tareas domésticas es el uso de una estrategia masculina que Bonino(2008)llama práctica de sobrecarga por omisión. Esta estrategia consiste en que los varones no indican explícitamente que a las mujeres les corresponde el rol de dueña de casa, sin embargo, no participan como ellas en la realización de dichas tareas. Esta situación genera un “desajuste entre el ideal igualitario y la realidad desigual, [situación que] provoca malestar, y [que] se expresa a través de conflictos en la pareja, unas veces explícitos y otras encubiertos, pero además genera en las mujeres sentimientos de resignación parcial y de amargura” (Dema, 2005, p. 153). La sobrecarga por omisión es una de las causas más frecuentes de conflictos y peleas en las parejas estudiadas:

"El otro tema como de conflicto es el orden. Los dos somos desordenados, a ninguno de los dos le gusta hacer aseo, pero yo me llevo más la carga” (I.P, Socióloga, 29 años).

En general lo que se aprecia es que la desigualdad en el reparto de las tareas domésticas sumada a diferentes formas de desacreditación utilizadas por parte de los varones se traduce en una de las maniobras de control y omisión en la participación de las tareas domésticas. Este mecanismo, que busca inferiorizar las opiniones o actitudes de las mujeres, se encontró en uno de los relatos de las entrevistadas con respecto a sus parejas pasadas; “Tú no sabes nada” obedece a aquella estrategia. Este medio de control hace que las mujeres duden de sí mismas, mermen su autoestima y se desvalorice, y a su vez, impide la comunicación dentro de la pareja ante el miedo de la reacción o comentarios del hombre, situación que dificulta la relación. El desigual reparto de tareas y en algunos casos la desautorización de las mujeres redundaba en una acentuación de los roles tradicionales de género sin que ellas se lo propusieran.

Sin embargo es importante recalcar que las entrevistadas no fueron pasivas ante estas prácticas micromachistas, por el contrario, respondieron activamente a esas inequidades y elaboraron distintas reacciones, actitudes y comportamientos para contrarrestar estas prácticas (Bonino, 2008). La mayoría puso en práctica mecanismos de resistencia basadas en la negociación, algunos de ellos explícitos (conversaciones y opiniones, donde los problemas de pareja de conversan y negocian) o implícitos, para contrarrestar prácticas de control.

"Pero siento que cada vez es más compartido [el orden de la casa]. Le ha costado si como asumir el rol pero yo he sido súper directa y a veces soy media bruta pa' (sic) decir las cosas, pero yo la otra vez le dije: yo no soy tu mamá y no soy tu nana, y no te voy a atender” (I.P, 29 años, socióloga).

Situación similar sucedía en el ámbito de la mantención económica del hogar, en la que había una negociación activa acerca de los asuntos económicos, y en algunas de las entrevistadas un esfuerzo constante por proteger sus propios ingresos ante la falta de recursos del varón; 
“A veces tenemos problemas de lucas (sic). Como [la pareja] una empresa pequeña y de repente quedan sin trabajo, hay un desfase, y claro, mi plata, era mi plata, que yo tenía guardada, pero eso lo solucionamos así cinco minutos, diez minutos, conversamos y ya, entonces hagamos esto y el Víctor: no, pero yo puedo hacer esto acá” (C.B, 38 años, socióloga).

Por su parte los mecanismos de resistencia adquirían notoriedad en los conflictos que tenían las parejas, como en el orden del hogar. Como se señaló respecto del reparto de tareas domésticas, los hombres evitaban involucrarse en este tipo de deberes generando así una sobrecarga de trabajo para ellas - además de tener un empleo remunerado, las entrevistadas debían ocupar sus tiempos de ocio trabajando en casa- lo que redundaba en quejas y reclamos por parte de las mujeres, siendo éstos últimos los mecanismos de resistencia más utilizado por las informantes. Pese a ello y al malestar manifestado, este tema no fue considerado como posible causal de ruptura, es más, algunas informantes le restaron importancia, situación que habla de un proceso de normalización de la desigualdad de género, aunque también, en algunos casos, era expresión del conflicto emocional que surge del desajuste entre la realidad y su ideal de pareja.

Estas estrategias funcionaron como un escudo para la defensa del ideal de pareja igualitaria. De hecho, con sus parejas ellas se constituyeron como las principales impulsoras de las negociaciones en pro de la continuación de dicho modelo:

"Yo creo que nunca peleamos, nunca. A veces yo me enojo por cosas así de aseo: no hiciste eso, le digo, y yo soy explosiva igual, entonces de repente le digo: aaahhh no hiciste la cuestión, y yo tengo que hacer todo, y me voy a lavar” (C.B, 38 años, socióloga).

Otra de las resistencias era la omisión. Lo mismo que hacían sus compañeros en cuanto a sobrecargarlas de trabajo doméstico a través de la omisión en la acción, ya sea a sabiendas de lo que hacen o no, ellas lo hacían conscientemente. Esta estrategia consistía en evitar el cumplimiento de las expectativas que el hombre tenía de ellas respecto a los roles de género tradicionales. Así, si la pareja esperaba que la mujer realizara tareas femeninas acorde al género (planchar, lavar, etc.) ella conscientemente decidía no hacerlo:

“Cuando no venía la Sra. Raquel [asesora del hogar], él se planchaba su ropa. Yo era una malvada, lo dejaba ahí, lo veía todo complicado. 'No pienso plancharle la ropa', pensada para mí, y el en el fondo de su corazón él esperaba que yo le planchara la camisa y yo: ¡qué pena, qué suerte que mi ropa no se plancha!” (M.M, 39 años, Contadora Auditora).

Las mujeres de la muestra valoran ante todo su independencia económica con respecto a sus parejas, inclusive más que de la relación en sí y 
la capacidad de decidir por sí mismas su destino. Es más, el significado que tienen dichos valores, las llevaron, muchas veces, a no claudicar en sus proyectos personales en pro del desarrollo de sus respectivos compañeros o de la continuidad de la vida en pareja. Por ello es que algunas informantes señalaron que, si bien tenían un proyecto de vida en común con sus compañeros y hasta podrían aceptar ciertas circunstancias desfavorables para ellas por la continuidad de la relación, (situaciones que no fuesen las "ideales”), no sacrificarían su desarrollo personal, y optarían siempre por sus propios proyectos vitales:

“A él [su pareja] le gustaría irse al campo, lo cual a mí no me molesta, porque al final él está acá soportando esta ciudad que no le gusta porque yo no me quiero ir, y lo hemos pensado, y yo digo: yo me voy al campo, no tengo problema, pero yo tengo que irme con todas las comodidades para allá, internet, porque yo puedo trabajar desde allá, si hago investigación por ejemplo, lo que hago acá, yo puedo hacerlo allá. Seguiría trabajando, no me gustaría irme a un lado y no trabajar o estar encargada de las gallinas, de las vacas” (C.B, 38 años, socióloga).

Todas las informantes han generado una trayectoria laboral desde temprana edad, lo que habla de una transición hacia identidades femeninas gestadas en lo público, lugar ocupado antes prácticamente en exclusividad por su par masculino. Este proceso ha sido un fenómeno que se autoimpulsa en el tiempo. Por lo mismo es que en la actualidad para las entrevistadas el trabajo remunerado recobra vital importancia, en tanto les otorga independencia económica con respecto del varón, libertad de movimiento y decisión, tanto de las actividades propias como en el hogar. Por lo mismo, más que la cantidad de dinero ganada, les interesa la posibilidad de usarlo libremente en actividades de elección propia o respecto de lo que quieren como destino. Por consiguiente, más que un fin, el trabajo remunerado es considerado por ellas como un medio que permite el acceso a diversas actividades que manifiestan como relevantes y que propicien su autonomía.

\section{Conclusiones}

La decisión de no tener hijos (as) se puede leer a priori como un indicador de emancipación y liberación femenina. De hecho la propia Simone de Beauvoir identificó en el matrimonio y la maternidad una de las principales causas de la subordinación de las mujeres, llamándolas abuscar la independencia económica como una forma de zafarse de la dependencia de los varones. Los tiempos han cambiado, no cabe duda que las mujeres han avanzado en materia de independencia económica, autonomía y protección jurídica. En ese sentido la revolución silenciosa de las mujeres ha resultado ser bastante más fructífera del siglo XX en términos de logros y conquistas femeninas. Sin embargo, no podemos afirmar alegremente que se ha superado la desigualdad o la inequidad de género. 
Ante la imposibilidad de deshacer los nudos críticos de la sociedad patriarcal muchas mujeres han diseñado consciente o inconscientemente estrategias de mantención de los espacios ganados de autonomía y han optado por la consecución de objetivos propios. Estos cambios se han traducido en una diversificación de las familiasHoy cada vez más éstas se constituyen de manera diversa, el matrimonio ha perdido importancia como forma hegemónica de unión, la maternidadse concibe progresivamente como opción antes que un mandato, se posterga y se va reduciendo paulatinamente el número de hijos (as) por mujer en pos de aprovechar la juventud en proyectos individuales y en la acumulación de bienestar material. En ese contexto la decisión por no tener hijos (as) se agrega de manera tímida al conjunto de transformaciones sociales y culturales de la sociedad chilena que nos hacen pensar en procesos de mayor igualdad de género o al menos su tránsito hacia dicho estado.

De acuerdo al estudio realizado las mujeres entrevistadas muestran una clara valoración de su autonomía, entendida como capacidad para decidir y ser dueñas de su propio destino. A partir de este valor que se conforma como un aspecto central de sus identidades, en conjunto con sus historias de vida, de acuerdo a lo cualidentificaron tempranamente en la maternidad y en el mandato asociado a él, el reparto desigual de tareas de acuerdo al género. Ese es un factor centralque identifican las entrevistadas como elemento que merma su autonomía. Por tanto se plantearon construir relaciones de pareja más igualitarias donde la decisión por no tener hijos es un elemento decisorio para conseguir dicho ideal. Los estudios universitarios, el trabajo y la administración de los recursos de manera independiente son elementos que coadyuvan a alcanzar los objetivos de igualdad en la relación de pareja.

Sin embargo los procesos sociales no van siempre en una sola dirección y a menudo son más bien contradictorios, es decir, de continuidad y cambio. El estudio realizado muestra que la opción de no tener hijos (as) puede ser leída como un potencial transformador de las relaciones de género porque es una decisión que va en contra de un mandato social fuertemente arraigado y esperado. En este sentido las mujeres que toman esta decisión, en consenso con sus parejas a través de los pactos fundantes, subvierten el precepto de la maternidad como la realización de toda mujer o destino femenino. Por tanto, podemos postular que dicha decisión contiene un ingrediente altamente liberador o emancipador que puede ser leído como un cambio social a nivel micro. De hecho la principal motivación que declararon las entrevistadas para no engendrar fue la posibilidad de establecer relaciones más igualitarias y evitar la sobrecarga de las labores asignadas por la división sexual del trabajo que habían vivido por los procesos de parentalización temprana. Sin embargo, la investigación muestra que la opción tomada no implicó necesariamente la desaparición de las relaciones de subordinación en tanto dentro de sus hogares mantienen una relación desigual con respecto al reparto de las tareas de orden y limpieza. En este sentido podemos identificar continuidades en las relaciones de género desigual, puesto que el hecho de no tener hijos no implica necesariamente 
transitar hacia relaciones más simétricas. En este punto las prácticas micromachistas son los elementos explicativos en la consecución de dichos objetivos. Pese a ello, un aspecto que nos indica la existencia de unatransición hacia relaciones más igualitarias en las mujeres del estudio esla búsqueda permanente de una relación más igualitaria expresada en actos de resistencia conscientes e inconscientes de las prácticas micromachistas, la importancia de la independencia económica y la defensa de la autonomía como dimensiones asociadas a estas estrategias y a la decisión de no tener hijos.

Como señalan investigaciones más recientes, el patriarcado mantiene su hegemonía, y se expresa en maniobras, estrategias sutiles y solapadas de machismos como los micromachismos al interior del hogar,que perpetúan la posición de poder de los varones. De alguna manera se cumple la expresión acuñada en la obra de Tomasi di Lampedusa, todo cambia para seguir igual. A pesar de que no es posible hacer una generalización a partir del trabajo realizado, podemos afirmar que la decisión de no tener hijos (as) persigue asegurar una relación distinta e igualitaria, al menos como aspiración en pos de ganar en autonomía. Las entrevistadas son conscientes de que se trata de una tarea constante que deben complementar con la independencia económica y administración propia del dinero, así como con constantes estrategias de resistencias que les recuerdan los mandatos de género sobre las labores domésticas. En este sentido podemos sostener que no sólo la decisión de no tener hijos (as), sino la consciencia de buscar las relaciones más igualitarias expresadas en la autonomía, la independencia económica y en un reparto igualitario de las tareas en el hogar, propician relaciones menos desiguales.

Por último el trabajo realizado nos invita a ahondar en decisiones que están en un recorrido similar a la decisión de no tener hijos (as), como son la opción de tener un solo hijo o la postergación de la maternidad a edades más adultas. De igual manera sería positivo replicar este trabajo con un grupo de referencia más amplio con la inclusión de los varones como sujetos de investigación. Estas alternativas nos permitirán despejar con más claridad los procesos de cambio en las relaciones de género que sabemos son lentos, pero que la historia ha demostrado no sin dificultades, que a menudo llegan para quedarse. 


\section{Notas}

${ }^{1}$ Este trabajo es una síntesis revisada y actualizada de la tesis de pregrado de Fernanda Chacón para optar al título de Socióloga titulada "Maternidad y cuerpo femenino en la Alta Modernidad. Análisis de los factores sociales que inciden en la decisión de no tener hijos en un grupo de mujeres profesionales de clase media”, Facultad de Ciencias Humanas, Universidad Arturo Prat, 2014 (Chacón, 2014)

${ }^{2}$ El orden patriarcal o el patrón de dominación patriarcal(Bastos, 1999; Bourdieu, 1999) se refiere de manera genérica a la supremacía de los varones con respecto de las mujeres en distintas dimensiones, una de esas dimensiones se refiere a la división sexual del trabajo por la cual se expresa un reparto desigual de tareas y actividades. De acuerdo a ello, las mujeres tienen a cargo las responsabilidades domésticas y el cuidado de los hijos e hijas, en el mercado de trabajo laboral se ubican en empleos más inestables y acceden a derechos desventajosos y remuneraciones menores, por ejemplo.

${ }^{3}$ En este tipo de diseño se recolectan "datos sobre las historias de vida y experiencias de determinadas personas para describirlas y analizarlas. Son de interés las personas en sí mismas y su entorno" (Salgado, 2007, p. 72) . Bajo este diseño se pueden analizar: la historia de vida del sujeto, el entorno en el cual vivió o vive y la secuencia de hechos y los resultados. Según Salgado (2007) bajo este diseño "el investigador reconstruye la historia de la persona o la cadena de sucesos (...), posteriormente los narra bajo su óptica y describe (sobre la base de la evidencia disponible) e identifica categorías y temas emergentes en los datos narrativos...”. (p.73)

${ }^{4}$ La primera de las acepciones, Childfree aparece por primera vez en 1913 en la Revista Americana de sociología, pero retoma fuerza en 1970 como alternativa al término Childless, cuyo significado - sin hijos (as)- está centrado más en la falta o ausencia que en la agencia, libertad de opciones y empoderamiento en las mujeres (Castaneda, 2013). Con respecto a NoMo (No Mother), esta sigla es acuñada en 2011 por Jody Day, y hace referencia no sólo a las mujeres que no desean tener hijos (as), sino también a las mujeres que por circunstancias ajenas a su voluntad no pudieron ser madres (problemas de fertilidad, por ejemplo) Ver: http://www.theguardian.com/ lifeandstyle/2012/feb/25/child-free-women-jody-day La última de las siglas es Dinks (DoubleIncome no kids), término anglosajón que engloba a las parejas de doble ingreso que voluntariamente deciden no tener hijos (as).

${ }^{5}$ La tasa de participación laboral femenina en Chile según el Instituto Nacional de Estadísticas para el trimestre Diciembre - Febrero 2014 llegó a un 48,7\%, cifra que si bien es histórica para el país, es bastante inferior al promedio de los países que integran la OECD, el que para el año 2011 era de un 62\% (OCDE, 2014).En relación con el acceso a la educación, según el Consejo Nacional de Educación, del total de matriculados el 2014 en las universidades, institutos profesionales, fuerzas armadas y centros de formación técnica, un 51\% corresponden a mujeres, superando por dos puntos porcentuales a los hombres (Consejo Nacional de Educación, 2014).

${ }^{6}$ La identidad es la articulación entre la subjetividad y la cultura, es decir, las imágenes que construimos de nosotros mismos están basadas también en elementos propios de la cultura en la que vivimos, donde se encuentran delimitados de antemano los comportamientos esperados para hombres y mujeres y las diferencias entre ellos - género-. La definición de lo que somos se construye en base a esas diferencias (Lamas, 1995). 
${ }^{7}$ Esfuerzos gubernamentales se están llevando a cabo para fomentar la responsabilidad del hombre en la crianza. El programa "Paternidad Activa" de Chile Crece Contigo es un claro ejemplo de ello. Ver: http://www.crececontigo.gob.cl/tag/paternidad-activa/

${ }^{8}$ La Sexta Encuesta Nacional UDP “Chile 2010: Percepciones y actitudes sociales”, resulta bastante reveladora al respecto; más de la mitad de los encuestados $(59,6 \%)$ está de Acuerdo con la afirmación de que el divorcio es la mejor solución cuando una pareja no puede resolver sus problemas matrimoniales, aceptación que aumenta entre los encuestados que tienen entre 18 y 45 años de edad, y que disminuye entre las personas que van desde los 45 y 61 años en adelante (De la Cea, 2011).

${ }^{9}$ Investigaciones llevadas a cabo en torno a los tiempos de productivos, reproductivos y de ocio de las parejas de doble ingreso, advierten que la llegada de los hijos (as) es uno de los factores más influyentes en la posición de desigualdad de las mujeres con respecto a sus parejas(Ajenjo y García Román, 2010). Como indica Ajenjo "la desigualdad incrementa considerablemente con la aparición del primer hijo, el cual para las mujeres significa una pérdida del tiempo productivo que es sustituido por tareas reproductivas, mientras que apenas afecta a los varones” (Ajenjo y García Román, 2010, p. 20). 


\section{Bibliografía}

Acker, J. (1990). Hierarchies, jobs, bodies: A theory of genderedorganizations. Gender and Society, 4 (2), 139-158.

Ajenjo, M., y García, J. (2010). El tiempo productivo, reproductivo y de ocio en las parejas de doble ingreso. Papers de Demografía, 355, 985-1006.

Altomonte, G., yAlbagly, C. (2010). No quiero tener hijos, Nunca. Recuperado dehttp://www.paula.cl/reportaje/no-quiero-tener-hijos-nunca/

Amorós, A. (1995). División sexual del trabajo. En C. Amorós (Ed.), 10 palabras claves sobre Mujer (pp. 257-295). Navarra, España: Verbo Divino.

Ariza, M., y Oliveira, O. (2001). Familias en transición y marcos conceptuales en redefinición. Papeles de Población, 7, (28), 9-39.

Baird, D. T., Collins, J., Evers, J. L. H., Leridon, H., Lutz, W., Velde, E. T., et al. (2010). Europethecontinentwiththelowestfertility. Human ReproductionUpdate, 16 (6), 590-602.

Barbosa, A., Segura, C., Garzón, D., y Parra, C. (2014). Significado de la experiencia del consumo de sustancias psicoactivas en un grupo de adolescentes institucionalizados. Avances en Psicología Latinoamericana, 32 (1), 53-69.

Bastos, S. (1999). ¿Una “patrón de dominación patriarcal” único e inmutable? En M. González de la Rocha (Ed.), Divergencias del modelo tradicional: Hogares de jefatura femenina en América Latina (pp. 38-75). México D.F, México: CIESAS/Conacyt.

Beck-Gernsheim, E. (2003). La reinvención de la familia: en busca de nuevas formas de convivencia. Barcelona: Editorial Paidós Ibérica.

Bonino, L. (1996). Micromachismos: La violencia invisible en la pareja. TheEuropeanProfeministmen's Network. Recuperado de http:// www.luisbonino.com/pdf/mM96.pdf

Bonino, L. (2008). Micromachismos - el poder masculino en la pareja 'moderna’. En J. A. Lozoya y J. C. Bedoya (Eds.) Voces de hombres por la igualdad. Recuperado de https://vocesdehombres. files.wordpress.com/2008/07/micromachismos-el-poder-masculinoen-la-pareja-moderna.pdf

Bosch, A., Amoroso, M.I. y Fernández, H. (2003). Arraigadas en la Tierra. En M.I. Amorosoet al., (Ed.), Malabaristas de la vida. Mujeres, tiempos y trabajos. Barcelona, España: Icaria. 
Bourdieu, P. (1999). La dominación masculina. Barcelona, España: Anagrama.

Caamaño. E. (2010). Mujer y trabajo: origen y ocaso del modelo del padre proveedor y la madre cuidadora. Revista de derecho (Valparaíso), (34), 179-209. https://dx.doi.org/10.4067/S0718-685120100 00100005

Camps, V. (1998). El siglo de las mujeres. Madrid, España: Ediciones Cátedra.

Cavenaghi, S. (2013). Fertiliy Decline and PublicPolicies to Address Population Rights: PerspectivefromLatinAmericaPopulationDivision. New York, UnitedStates: UnitedNationsDepartment of Economic and Social Affairs.

Centro Alemán de Información. (2013). Mujeres sin hijos. Recuperado de http://www.alemaniaparati.diplo.de/Vertretung/mexiko-dz/es/05 Sociedad/Poblacion/Mujeressinhijos.html Consultado el: 21 de julio de 2016

CEPAL. (2008). Fecundidad. Observatorio $N^{\circ}$ 5. Recuperado de http:// www.cepal.org/cgi-bin/getProd.asp?xml=/publicaciones/xml/8/36498/ P36498.xml\&xsl=/celade/tpl/p9f.xsl\&base=/tpl/top-bottom.xslt

Code, L. (1991). What Can SheKnow? FeministTheory and theConstruction of Knowledge. Ithaca, UnitedState: CornellUniversityPress.

Consejo Nacional de Educación. (2014). Índices: Estadísticas y Bases de Datos. Pregrado. Matrícula por sexo. Recuperado de http:// www.cned.cl/

Coria, C. (1991). El sexo oculto del dinero. Formas de dependencia femenina. Barcelona, España: Paidós.

De la Cea. (2011). Chile 2010. Sexta Encuesta Nacional UDP. Recuperado de http://encuesta.udp.cl/publicaciones/

Dema, S. (2005). Entre la tradición y la modernidad: las parejas españolas de doble ingreso. Papers: Revista de Sociología(77) 135-155.doi: http://dx.doi.org/10.5565/rev/papers/v77n0.934

Dema, S. (2006). Una pareja, dos salarios. El dinero y las relaciones de poder en las parejas de doble ingreso. Madrid, España: CIS.

Díaz, D., Godoy, L., yStecher, A. (2005). Significados del trabajo, identidad y ciudadanía. La experiencia de hombres y mujeres en un mercado laboral flexible. Santiago, Chile: CEM 
Duby, G., Perrot, M., Thébaud, F., y Nash, M. (2006). Historia de las mujeres en Occidente.Madrid, España: Taurus.

Friedman, M. (2014). RelationalAutonomy and Independence. In A. Vetman\& M. Piper (Eds.), Autonomy, Oppression and Gender. Oxford, England: Oxford UniversityPress

García, B., y Oliveira, O. (2007). Trabajo extradoméstico y relaciones de género: Una nueva mirada. En I. Brighentiy M. Enghel (Eds.).Género, familias y trabajo: rupturas y continuidades. Desafíos para la investigación política (pp. 49-87). Buenos Aires, Argentina: CLACSO

Gonzálvez, H. (2015). Los estudios de familia en Chile. Características y desafíos para el futuro. En H. Gonzálvez (Ed.), Diversidades familiares, cuidados y migración. Nuevos enfoques y viejos dilemas (pp. 17-37). Santiago, Chile: Ediciones Universidad Alberto Hurtado.

Hird, M., \&Abshoff, K. (2000). Womenwithoutchildren: A contradiction in terms? Journal of comparativefamilystudies, 31 (3), 347-366.

Hooper, M., Wallace, S., Doehler, K., \&Dantzler, J. (2012). Parentification, ethnicidentity, and psychologicalhealth in Black and White American collegestudents: implications of family-of-origin and cultural factors. Journal of ComparativeFamilyStudies, 43 (6), 811-835.

INE. (2006). Fecundidad en Chile. Situación reciente. Recuperado de http:/ /www.ine.cl/canales/chile_estadistico/demografia_y_vitales/ demografia/pdf/fecundidad.pdf

INE. (2011). Evolución de la fecundidad en Chile 1990-2011.Recuperado de http://www.ine.cl/canales/menu/publicaciones/calendario_ de_publicaciones/pdf/info_fecundidad.pdf

Jaggar, A. (1985). FeministPolitics and Human Nature. Totowa, UnitedStates: Rowman and Allanheld.

Jelin, E. (2006). Pan y afectos. La transformación de las familias. México D. F, México: Fondo de Cultura Económica.

Jelin, E., y Paz, G. (1991). Familia, género en América Latina: Cuestiones históricas y contemporáneas. Buenos Aires, Argentina: Centro de Estudios de Estado y Sociedad.

Kandiyoti, D. (1988). BargainingwithPatriarchy. Gender and Society, 2 (3), 274-290.

Lagarde, M. (1999). Claves feministas para el poderío y la autonomía de las mujeres. Sevilla, España: Instituto Andaluz de la Mujer. 
Lamas, M. (1995). Cuerpo e Identidad. En M. Arango, M. León y L. Viveros (Eds.), Género e Identidad. Ensayos sobre lo masculino y lo femenino (pp. 61-82). Bogotá, Colombia: Tercer Mundo Editores.

Mackenzie, C. (2014). ThreeDimensions of Autonomy: A RelationalAnalysis. In A. Veltman\& M. Piper (Eds.), Autonomy, Oppression and Gender, Oxford, England: Oxford UniversityPress.

Mensch, B., Sing, S., \&Casterline, J. (2005). Trends in theTiming of FirstMarriageAmongMen and Women in theDevelopingWorld. PolicyResearchDivisionWorkingPapers (202). Recuperado de http:/ /www.popcouncil.org/uploads/pdfs/wp/202.pdf

Molina, C. (2003). Género y poder desde sus metáforas. Apuntes para una topografía del patriarcado. En S. E. Tubert (Ed.), Del sexo al género. Los equívocos de un concepto (pp. 123-159). Madrid, España: Cátedra.

Moore, H. L. (2004). Antropología y feminismo. Madrid, España: Cátedra.

OCDE. (2014). Panorama económico de la OCDE. Recuperado de http:// www.oecd.org/centrodemexico/estadisticas/

Olavarría, J. (2014). Transformaciones de la familia conyugal en Chile en el período de la transición democrática (1990-2011). Polis, Revista Latinoamericana13 (37), 473-497. Recuperado de http:// polis.revues.org/9973

ONU. (2015). WorldFertilityPatterns 2015. Recuperado de http:// www.un.org/en/development/desa/population/publications/pdf/ fertility/world-fertility-patterns-2015.pdf

Parella, S. (2003). Mujer, migrante y trabajadora: la triple discriminación. Barcelona, España: Anthropos.

Quijada, S. (2010). Caracterización de la población que decide tener o no hijos en el futuro. Recuperado de http://estudios.sernam.cl/documentos/?eMTgzNDA0MQ==-Caracterizaci\%C3\%B3n_de_la_poblaci\% C3\%B3n_que_decide_tener_o_no_hijas/os_en_el_futuro._.Consultado el:

Salazar, G. (1992). La mujer de “bajo pueblo”en Chile: bosquejo histórico. Proposiciones, (21), 89-107.

Saletti, L. (2008). Propuestas teóricas feministas en relación al concepto de maternidad. Clepsydra, 7, 169-183.

Salgado, A. C. (2007). Investigación cualitativa: diseños, evaluación del rigor metodológico y retos. Liberabit, 13, 71-78. 
Salvo, I., yGonzálvez, H. (2015). Monoparentalidades electivas en Chile: Emergencias, tensiones y perspectivas. Psicoperspectivas. Individuo y Sociedad, 14 (2), 40-50. Recuperado de http:// www.psicoperspectivas.cl/index.php/psicoperspectivas/article/view/ 541

Stacey, J., \&Thorne, B. (1985). Themissingfeministrevolution in sociology. Social Problems, 32 (4), 301-317.

Tapia, M. (2010). Yo venía con un sueño... Relaciones de género entre inmigrantes de origen boliviano en Madrid 2000-2007. Madrid, España: Universidad Complutense/Instituto de Investigación José Ortega y Gasset.

Tobío, C. (2005). Madres que trabajan. Dilemas y estrategias. Madrid, España: Cátedra.

USAID. (2014). WorldPopulation Data SheetRecuperado dehttp:// www.prb.org/pdf14/2014-world-population-data-sheet_eng.pdf

Valcárcel, A. (2009). Feminismo en el mundo global. Madrid, España: Cátedra

Vogler, C. (1998). Money in thehousehold: someunderlingsissues of power. SociologicalReview, 46 (4), 687-713.

Vogler, C., \&Pahl. (1999). Money, power and inequality. In A. Graham (Ed.), Thesociology of thefamily: a reader. Oxford, England: Malden.

Recibido: 21.09.15

Aceptado: 26.07.16 